\title{
Baker-Akhiezer Modules on Rational Varieties
}

Irina A. MELNIK ${ }^{\dagger}$ and Andrey E. MIRONOV ${ }^{\ddagger}$

$\dagger$ Novosibirsk State University, 630090 Novosibirsk, Russia

E-mail: sibirochka@ngs.ru

$\ddagger$ Sobolev Institute of Mathematics, 630090 Novosibirsk, Russia

E-mail:mironov@math.nsc.ru

URL: http://math.nsc.ru/LBRT/d6/mironov/

Received January 05, 2010, in final form April 03, 2010; Published online April 07, 2010

doi:10.3842/SIGMA.2010.030

\begin{abstract}
The free Baker-Akhiezer modules on rational varieties obtained from $\mathbb{C} P^{1} \times$ $\mathbb{C} P^{n-1}$ by identification of two hypersurfaces are constructed. The corollary of this construction is the existence of embedding of meromorphic function ring with some fixed pole into the ring of matrix differential operators in $n$ variables.
\end{abstract}

Key words: commuting differential operators; Baker-Akhiezer modules

2010 Mathematics Subject Classification: 14H70; 35P30

\section{Introduction}

The Baker-Akhiezer modules (BA-modules) over the ring of differential operators were introduced by Nakayashiki (see $[1,2]$ ). These modules are constructed on the basis of spectral data which include an algebraic variety $X$ and some additional objects. In the one-dimensional case the module elements are the usual Baker-Akhiezer functions.

The BA-module $M$ consists of functions $\psi(x, P)$ which depend on $x \in \mathbb{C}^{n}$, where $n=\operatorname{dim}_{\mathbb{C}} X$ and $P \in X$. If $x$ is fixed, then the function $\psi$ is the section of a bundle over $X$, and $\psi$ has an essential singularity on divisor $Y \subset X$. The elements $\psi \in M$ have the following properties:

- $\partial_{x_{j}} \psi \in M$ and $f(x) \psi \in M$, where $f(x)$ is an analytical function in a neighbourhood of a fixed point $x_{0}$;

- if $\lambda$ is a meromorphic function with a pole on $Y$, then $\lambda \psi \in M$.

These properties mean that $M$ is the module over the ring of differential operators $\mathcal{D}_{n}=$ $\mathcal{O}\left[\partial_{x_{1}}, \ldots, \partial_{x_{n}}\right]$, where $\mathcal{O}$ is the ring of analytical functions in a neighbourhood of $x_{0}$, and over the ring $A_{Y}$ of meromorphic functions on $X$ with the pole on $Y$.

The finitely generated free BA-modules over $\mathcal{D}_{n}$ are of the main interest as in this case the construction allows to build commutative rings of differential operators. Let us choose the basis $\psi_{1}(x, P), \ldots, \psi_{N}(x, P)$ in $M$. Let $\Psi(x, P)$ denote vector-function $\left(\psi_{1}(x, P), \ldots, \psi_{N}(x, P)\right)^{\top}$. Then for $\lambda \in A_{Y}$ there is only one differential operator $D(\lambda)$ with $N \times N$-matrix coefficients such that

$$
D(\lambda) \Psi(x, P)=\lambda(P) \Psi(x, P) .
$$

The operators $D(\lambda)$ and $D(\mu)$ obviously commutate with each other for different $\lambda$ and $\mu \in A_{Y}$. Thus, the considered construction makes it possible to obtain solutions of nonlinear differential equations which are equivalent to the condition of commutation of differential operators.

The following examples of the free BA-modules over $\mathcal{D}_{n}$ are known. In [1] and [2] it is shown that the BA-modules on Abelian varieties are free under some restrictions on spectral data. 
In [2] it is also shown that the restriction of the BA-module from three-dimensional Abelian variety to the shifted theta-divisor remains free (over the ring of differential operators of two variables).

In [3] it is shown ${ }^{1}$ that the restriction of BA-module from Abelian variety to the complete intersection of the shifted theta-divisors remains free. On these grounds we found the solutions of multidimensional analog of Kadomtsev-Petviashvili hierarchy

$$
\left[\partial_{t_{k}}-A_{k}, \partial_{t_{m}}-A_{m}\right]=0
$$

where $A_{k}$ and $A_{m}$ are matrix differential operators of $n$ variables.

In [4] and [5] the sufficient conditions for the spectral data, which correspond to the free BA-modules were found (see Theorem 4.1 in [4] and Theorem 3.3 in [5]). It is not clear how to find the algebraic varieties, satisfying these conditions. In [4] and [5] there are two examples satisfying these conditions. In [5] sufficient conditions for spectral data corresponding to the solutions of the equation (1) were ascertained; in the paper a corresponding example was also demonstrated.

Note that in all the examples above the construction is either implicit (see [4] and [5]), or the solutions are expressed in terms of theta-functions (see [1, 2], and [3]).

Those who wish to read more widely in the theory of commuting operators of several variables and BA-modules can turn to [6].

The aim of this paper is to demonstrate the construction of Nakayashiki for the rational varieties. For the rational spectral variety, the BA-module elements and coefficients of commutating differential operators are expressed in terms of elementary functions.

Our initial idea was to obtain BA-modules on rational varieties from BA-modules of Nakayashiki by degenerating of Abelian varieties in the same way as soliton solutions of $\mathrm{KdV}$ are obtained from finite-gap solutions by degenerating of smooth spectral curves to spheres with double points. We considered many candidates of rational varieties and, as a result, we found varieties $\Gamma$ and $\Omega$ (see below) appropriate for our goals.

In the next section we describe the spectral data used in this paper and formulate our main results. In Sections 3 and 4 we show that the BA-modules on $\Gamma$ and $\Omega$ are free. In Section 5 we present explicit examples of commutating operators. In the Appendix we show that on $\Gamma$ and $\Omega$ there are structures of algebraic varieties (it not follows directly from the definition of $\Gamma$ and $\Omega$ ).

\section{Main results}

Let us fix $a_{1}, a_{2}, b_{1}, b_{2} \in \mathbb{C}$ such that $\left(a_{i}, b_{i}\right) \neq(0,0)$ and $\left(a_{1}: b_{1}\right) \neq\left(a_{2}: b_{2}\right)$. Let us also fix nondegenerate linear map $\mathcal{P}: \mathbb{C}^{n} \rightarrow \mathbb{C}^{n}$. This map induce the map $\mathbb{C} P^{n-1} \rightarrow \mathbb{C} P^{n-1}$, which we denote by the same symbol $\mathcal{P}$. Let $\Gamma$ denote the variety constructed from $\mathbb{C} P^{1} \times \mathbb{C} P^{n-1}$ by identification of two hypersurfaces

$$
p_{1} \times \mathbb{C} P^{n-1} \sim p_{2} \times \mathbb{C} P^{n-1}
$$

with the use of $\mathcal{P}$, where $p_{i}=\left(a_{i}: b_{i}\right)$. Namely, let us identify

$$
\left(a_{1}: b_{1}, t\right) \sim\left(a_{2}: b_{2}, \mathcal{P}(t)\right), \quad t=\left(t_{1}: \cdots: t_{n}\right) \in \mathbb{C} P^{n-1} .
$$

Let $f(P)$ be the following function on $\mathbb{C}^{n+2}$

$$
f\left(z_{1}, z_{2}, t_{1}, \ldots, t_{n}\right)=\sum_{i=1}^{n}\left(\alpha_{i} z_{1} t_{i}+\beta_{i} z_{2} t_{i}\right), \quad \alpha_{i}, \beta_{i} \in \mathbb{C} .
$$

\footnotetext{
${ }^{1}$ In the proof of freeness of the BA-modules in [3] there is a gap, an additional proposition is required. The complete proof will appear in the work of K. Cho, A. Mironov, and A. Nakayashiki "Baker-Akhiezer module on the intersection of shifted theta divisors" (submitted to Publ. RIMS).
} 
such that the following identity takes place

$$
f\left(a_{1}, b_{1}, t\right)-A f\left(a_{2}, b_{2}, \mathcal{P}(t)\right)=0
$$

for fixed $A \in \mathbb{C}^{*}$ and every $t=\left(t_{1}, \ldots, t_{n}\right)$. Identity (3) gives the restriction on the choice of $\alpha_{i}, \beta_{i}$. According to (3), the equation

$$
f\left(z_{1}: z_{2}, t_{1}: \cdots: t_{n}\right)=0
$$

correctly defines a hypersurface in $\Gamma$.

We denote eigenvalues and eigenvectors of $\mathcal{P}$ by $\lambda_{j}$ and $\mathbf{w}_{j}$ respectively. Henceforth, we assume that

$$
\lambda_{j} \neq \lambda_{k} \quad \text { at } \quad j \neq k
$$

and $f(P)$ is chosen such that

$$
f\left(a_{1}, b_{1}, \mathbf{w}_{j}\right) \neq 0, \quad j=1, \ldots, n .
$$

We introduce $n$ functions on $\mathbb{C}^{n+2}$

$$
f_{i}\left(z_{1}, z_{2}, t_{1}, \ldots, t_{n}\right)=\sum_{k=1}^{n}\left(\alpha_{i k} z_{1} t_{k}+\beta_{i k} z_{2} t_{k}\right)
$$

such that $f_{i}$ satisfies the identity:

$$
\frac{f_{i}\left(a_{1}, b_{1}, t\right)}{f\left(a_{1}, b_{1}, t\right)}-\frac{f_{i}\left(a_{2}, b_{2}, \mathcal{P}(t)\right)}{f\left(a_{2}, b_{2}, \mathcal{P}(t)\right)}-c_{i}=0, \quad c_{i} \in \mathbb{C}
$$

for every $t=\left(t_{1}, \ldots, t_{n}\right) \in \mathbb{C}^{n}$. By $(3)$, this identity is equivalent to

$$
f_{i}\left(a_{1}, b_{1}, t\right)-A f_{i}\left(a_{2}, b_{2}, \mathcal{P}(t)\right)-c_{i} f\left(a_{1}, b_{1}, t\right)=0 .
$$

The dimension of the space of these functions is equal to $(n+1)$. We choose $f_{1}, \ldots, f_{n}$ such that $f_{1}, \ldots, f_{n}$ and $f$ are linearly independent. Moreover, we choose parameters $(\alpha, \beta),\left(\alpha_{i}, \beta_{i}\right)$ in a general position, that means that the parameters belong to some open everywhere dense domain (more precisely, such that equation (18) has no multiple solutions).

Let us fix $\Lambda \in \mathbb{C}$. Let

$$
M_{\Gamma}(k)=\left\{\psi(x, P)=\frac{h\left(x_{1}, \ldots, x_{n}, P\right)}{f^{k}(P)} \exp \left(\sum_{j=1}^{n} \frac{f_{j}(P)}{f(P)} x_{j}\right)\right\},
$$

where

$$
\psi\left(x, a_{1}: b_{1}, t\right)-\Lambda \psi\left(x, a_{2}: b_{2}, \mathcal{P}(t)\right)=0
$$

for $t=\left(t_{1}: \cdots: t_{n}\right) \in \mathbb{C} P^{n-1}$. Here $P=\left(z_{1}: z_{2}, t\right) \in \mathbb{C} P^{1} \times \mathbb{C} P^{n-1}$ and $h(x, P)$ has the form

$$
h(x, P)=\sum_{0 \leq j \leq k,|\alpha|=k} h_{j \alpha}(x) z_{1}^{j} z_{2}^{k-j} t^{\alpha},
$$

where $\alpha=\left(\alpha_{1}, \ldots, \alpha_{n}\right), t^{\alpha}=t_{1}^{\alpha_{1}} \cdots t_{n}^{\alpha_{n}}$.

According to (8), if $\psi \in M_{\Gamma}(k)$, then $\partial_{x_{j}} \psi \in M_{\Gamma}(k)$. Consequently, we have $n$ mapping

$$
\partial_{x_{j}}: M_{\Gamma}(k) \rightarrow M_{\Gamma}(k+1), \quad j=1, \ldots, n .
$$


Thereby, on the set

$$
M_{\Gamma}=\bigcup_{k=1}^{\infty} M_{\Gamma}(k)
$$

the structure of the BA-module over the ring of differential operators $\mathcal{D}_{n}=\mathcal{O}\left[\partial_{x_{1}}, \ldots, \partial_{x_{n}}\right]$ is defined.

Theorem 1. $M_{\Gamma}$ is a free $\mathcal{D}_{n}$-module of rank $n$ generated by $n$ functions from $M_{\Gamma}(1)$.

Corollary 1. There is a ring embedding

$$
D: A_{f} \rightarrow \operatorname{Mat}\left(n, \mathcal{D}_{n}\right)
$$

of the rings of meromorphic functions on $\Gamma$ with the poles on hypersurface $f=0$ into the ring of differential operators in variables $x_{1}, \ldots, x_{n}$ with the matrix coefficients of size $n \times n$.

For $n=2$ it is possible to consider another way of identification of two curves in $\mathbb{C} P^{1} \times \mathbb{C} P^{1}$. Let $\Omega$ denote the variety which comes out from $\mathbb{C} P^{1} \times \mathbb{C} P^{1}$ by the identification of two lines

$$
p_{1} \times \mathbb{C} P^{1} \sim \mathbb{C} P^{1} \times p_{2} .
$$

Videlicet, we identify the following points:

$$
\left(p_{1}, t\right) \sim\left(\mathcal{P}(t), p_{2}\right)
$$

where $p_{i}, t \in \mathbb{C} P^{1}$. We assume that $\mathcal{P}\left(p_{2}\right) \neq p_{1}$. Therefore, in an appropriate coordinate system, the variety $\Omega$ has the form

$$
\Omega=\mathbb{C} P^{1} \times \mathbb{C} P^{1} /\left\{\left(1: 0, t_{1}: t_{2}\right) \sim\left(t_{1}: t_{2}, 0: 1\right)\right\} .
$$

Indeed, on $\mathbb{C} P^{1} \times \mathbb{C} P^{1}$ we make the following change of coordinates:

$$
\left(z^{\prime}, w^{\prime}\right)=(z, \mathcal{P}(w))
$$

where $(z, w),\left(z^{\prime}, w^{\prime}\right)$ are the old and the new coordinates on $\mathbb{C} P^{1} \times \mathbb{C} P^{1}$ respectively. Then $\Omega$ is obtained by the identification of the points:

$$
\left(p_{1}, \mathcal{P}(t)\right) \sim\left(\mathcal{P}(t), \mathcal{P}\left(p_{2}\right)\right)
$$

Now on each of components $\mathbb{C} P^{1} \times \mathbb{C} P^{1}$ we do the same change of coordinates such that the points $p_{1}$ and $\mathcal{P}\left(p_{2}\right)$ in the new system have coordinates $(1: 0)$ and $(0: 1)$ respectively. In the new coordinates the variety $\Omega$ has the form (10).

Let $g$ denote the following function

$$
g\left(z_{1}, z_{2}, w_{1}, w_{2}\right)=\alpha z_{1} w_{1}+\beta z_{1} w_{2}+\gamma z_{2} w_{1}+\delta z_{2} w_{2}, \quad \alpha, \beta, \gamma, \delta \in \mathbb{C}
$$

such that for $\left(t_{1}, t_{2}\right) \in \mathbb{C}^{2}$ the following identity is fulfilled

$$
g\left(1,0, t_{1}, t_{2}\right)-B g\left(t_{1}, t_{2}, 0,1\right)=0,
$$

where $B \in \mathbb{C}^{*}$ is fixed. We assume that

$$
g(0,1,0,1) \neq 0
$$


We introduce two more functions

$$
g_{i}\left(z_{1}, z_{2}, w_{1}, w_{2}\right)=\alpha_{i} z_{1} w_{1}+\beta_{i} z_{1} w_{2}+\gamma_{i} z_{2} w_{1}+\delta_{i} z_{2} w_{2}, \quad \alpha_{i}, \beta_{i}, \gamma_{i}, \delta_{i} \in \mathbb{C}, \quad i=1,2
$$

such that for $\left(t_{1}, t_{2}\right) \in \mathbb{C}^{2}$ the identity is fulfilled

$$
\frac{g_{i}\left(1,0, t_{1}, t_{2}\right)}{g\left(1,0, t_{1}, t_{2}\right)}-\frac{g_{i}\left(t_{1}, t_{2}, 0,1\right)}{g\left(t_{1}, t_{2}, 0,1\right)}-c_{i}=0, \quad c_{i} \in \mathbb{C} .
$$

The dimension of the space of such functions is equal to 3. According to (11), the identity (12) is equivalent to

$$
g_{i}\left(1,0, t_{1}, t_{2}\right)-B g_{i}\left(t_{1}, t_{2}, 0,1\right)-c_{i} g\left(1,0, t_{1}, t_{2}\right)=0 .
$$

Let us choose $g_{1}$ and $g_{2}$ in such a way that $g_{1}, g_{2}$ and $g$ are linearly independent and the under radical expression in (23) does not vanish (this always can be achieved by the infinitesimal changes of $c_{1}$ and $c_{2}$ ). Let

$$
G_{1}(P)=\frac{g_{1}(P)}{g(P)}, \quad G_{2}(P)=\frac{g_{2}(P)}{g(P)} .
$$

Let us fix $\Lambda \in \mathbb{C}$. By $M_{\Omega}(k)$ we denote the set of functions of the form

$$
M_{\Omega}(k)=\left\{\varphi=\frac{\tilde{h}(x, y, P)}{g^{k}(P)} \exp \left(x G_{1}(P)+y G_{2}(P)\right)\right\}
$$

for which the identity

$$
\varphi\left(x, y, 1: 0, t_{1}: t_{2}\right)-\Lambda \varphi\left(x, y, t_{1}: t_{2}, 0: 1\right)=0
$$

is fulfilled, where $\tilde{h}$ is the function of the form (9).

Let

$$
M_{\Omega}=\bigcup_{k=1}^{\infty} M_{\Omega}(k)
$$

$M_{\Omega}$ is the module over $\mathcal{D}=\mathcal{O}\left[\partial_{x}, \partial_{y}\right]$.

Theorem 2. $M_{\Omega}$ is a free $\mathcal{D}$-module of the rank 2 generated by two functions from $M_{\Omega}(1)$.

Let $A_{g}$ denote the ring of the meromorphic functions on $\Omega$ with the pole on the curve defined by the equation $g(P)=0$.

Corollary 2. There is a ring embedding

$$
D: A_{g} \rightarrow \operatorname{Mat}(2, \mathcal{D})
$$

of $A_{g}$ into the ring of $2 \times 2$-matrix differential operators in variables $x$ and $y$.

Remark 1. To be more precise, the freeness of $M_{\Gamma}$ and $M_{\Omega}$ is a corollary of the fact that corresponding graded grD-modules are free, where the graduation is induced by the degree of the operators and the order of poles respectively. Below we virtually prove the freeness of graded modules. 


\section{Proof of Theorem 1}

\subsection{Combinatorial structure of $M_{\Gamma}$}

We find the rank of the module $M_{\Gamma}(k)$ over $\mathcal{O}$. The dimension of the space of functions $\{h(x, P)\}$ (see (9)) is equal $C_{k+1}^{1} C_{k+n-1}^{n-1}$ (for fixed $x$ ). The condition (8) with the help of (3) and (6) can be written in the equivalent form

$$
h\left(x, a_{1}, b_{1}, t\right)-h\left(x, a_{2}, b_{2}, \mathcal{P}(t)\right) \Lambda A^{k} e^{-c x}=0,
$$

where $c x=\sum_{j=1}^{n} c_{j} x_{j}$. This equality means that the coefficients of the homogeneous polynomial in $t_{1}, \ldots, t_{n}$ of the degree $k$, situated in the left part, are equal to 0 . It gives $C_{k+n-1}^{n-1}$ restrictions on the choice of coefficients of $h(x, P)$. Thereby,

$$
\operatorname{rank}_{\mathcal{O}} M_{\Gamma}(k)=(k+1) C_{k+n-1}^{n-1}-C_{k+n-1}^{n-1}=k C_{k+n-1}^{n-1} .
$$

Let $\mathcal{D}_{n}^{k-1}$ be the differential operators of order $k-1$ at most. We have

$$
\operatorname{rank}_{\mathcal{O}} \mathcal{D}_{n}^{k-1}=C_{k+n-1}^{n}=\frac{(k+n-1) !}{(k-1) ! n !}=\frac{k(k+1) \cdots(k+n-1)}{n !}=\frac{k}{n} C_{k+n-1}^{n-1} .
$$

Comparing (13) and (14), we can expect that $M_{\Gamma}$ is a free module of the rank $n$ generated by $n$ functions from $M_{\Gamma}(1)$.

\subsection{Module $N$}

Let us choose $n$ functions $\psi_{1}, \ldots, \psi_{n} \in M_{\Gamma}(1)$ independent over $\mathcal{O}$

$$
\psi_{k}(x, P)=\frac{h_{k}(x, P)}{f(P)} \exp \left(\sum_{j=1}^{n} \frac{f_{j}(P)}{f(P)} x_{j}\right),
$$

where

$$
h_{k}(x, P)=\sum_{i=1}^{n}\left(h_{k i}^{1}(x) z_{1} t_{i}+h_{k i}^{2}(x) z_{2} t_{i}\right) .
$$

Consider the module $N$ over $\mathcal{D}_{n}$ generated by the functions $\psi_{1}, \ldots, \psi_{n}$

$$
N=\left\{\sum_{i=1}^{n} d_{i} \psi_{i} \mid d_{i} \in \mathcal{D}_{n}\right\} .
$$

We show that the module $N$ is free (Lemma 1) and as a consequence from the combinatorial calculation we get that the modules $M_{\Gamma}$ and $N$ coincide (Lemma 3).

Lemma 1. $N$ is a free $\mathcal{D}_{n}$-module of rank $n$.

Proof. Suppose that the assertion is not true, i.e. there are differential operators $d_{1}, \ldots, d_{n} \in$ $\mathcal{D}_{n}$ such that

$$
d_{1} \psi_{1}+\cdots+d_{n} \psi_{n}=0
$$

where

$$
d_{j}=\sum_{\alpha:|\alpha| \leq K} a_{j \alpha}(x) \partial_{x}^{\alpha},
$$

$K$ is maximal order of operators $d_{j}$. 
Let us divide (15) by $\exp \left(\sum \frac{f_{j}}{f} x_{j}\right)$, multiply by $f^{K+1}$ and restrict the received equality on the hyperspace $f(P)=0$. We receive the following equality (for the compactness of the record the arguments are skipped)

$$
\begin{aligned}
& h_{1}\left(a_{1(K, 0, \ldots, 0)} f_{1}^{K}+a_{1(K-1,1,0, \ldots, 0)} f_{1}^{K-1} f_{2}+\cdots+a_{1(0, \ldots, 0, K)} f_{n}^{K}\right)+\cdots \\
& \quad+h_{n}\left(a_{n(K, 0, \ldots, 0)} f_{1}^{K}+a_{n(K-1,1,0, \ldots, 0)} f_{1}^{K-1} f_{2}+\cdots+a_{n(0, \ldots, 0, K)} f_{n}^{K}\right)=0 .
\end{aligned}
$$

The hypersurfaces

$$
f(P)=0, \quad f_{j}(P)=0, \quad j=1, \ldots, n, \quad j \neq k .
$$

$\left(f_{k}(P)=0\right.$ is left out) have $n$ points of intersections. Indeed, let us consider (17) as linear equations in $t_{1}, \ldots, t_{n}$. These equations have nonzero solutions if the determinant $\Delta_{j}$, composed from coefficients (coefficients are linear forms $\alpha_{i} z_{1}+\beta_{i} z_{2}$ or $\alpha_{s i} z_{1}+\beta_{s i} z_{2}$ ), equals 0

$$
\Delta_{j}=0
$$

So, (18) is a homogeneous equation in $z_{1}, z_{2}$ of degree $n$, and by our assumption has no multiple solutions. By $P_{i}^{k}, i=1, \ldots, n$ we denote the intersection points of hypersurfaces (17).

Let us substitute $P_{i}^{n}$ in (16), divide all equalities by $f_{n}^{K}\left(f_{n}\left(P_{i}^{n}\right) \neq 0\right.$, see Lemma 4$)$. We obtain a linear system of equations on coefficients $a_{k(0, \ldots, 0, K)}$ of operators $d_{k}$

$$
\begin{aligned}
& h_{1}\left(x, P_{1}^{n}\right) a_{1(0, \ldots, 0, K)}+\cdots+h_{n}\left(x, P_{1}^{n}\right) a_{n(0, \ldots, 0, K)}=0, \\
& \ldots \ldots \ldots \ldots \ldots \ldots \ldots \ldots \ldots \ldots \ldots \ldots \ldots \ldots \ldots \ldots \ldots \ldots \\
& h_{1}\left(x, P_{n}^{n}\right) a_{1(0, \ldots, 0, K)}+\cdots+h_{n}\left(x, P_{n}^{n}\right) a_{n(0, \ldots, 0, K)}=0 .
\end{aligned}
$$

We need

\section{Lemma 2. The inequality holds}

$$
\operatorname{det}\left(\begin{array}{ccc}
h_{1}\left(x, P_{1}^{k}\right) & \ldots & h_{n}\left(x, P_{1}^{k}\right) \\
\vdots & \ddots & \vdots \\
h_{1}\left(x, P_{n}^{k}\right) & \ldots & h_{n}\left(x, P_{n}^{k}\right)
\end{array}\right) \neq 0 .
$$

The proof of this lemma is given in Subsection 3.3.

By Lemma 2, the solution of the system (19) is

$$
a_{1(0, \ldots, 0, K)}=0, \quad \ldots, \quad a_{n(0, \ldots, 0, K)}=0 .
$$

Similarly, for $k=1, \ldots, n-1$ from $\operatorname{det}\left(h_{j}\left(x, P_{i}^{k}\right)\right) \neq 0$ it follows that the coefficients $a_{j(0 \ldots K \ldots 0)}$ must vanish ( $K$ is on the $k$-th place).

To show that the coefficients $a_{j(0, \ldots 0, m, l)}$, where $m+l=K$, vanish we should restrict (16) on $f(P)=0, f_{1}(P)=0, \ldots, f_{n-2}(P)=0$

$$
\begin{aligned}
& h_{1}\left(a_{1(0, \ldots, 0, K-1,1)} f_{n-1}^{K-1} f_{n}+\cdots+a_{1(0, \ldots, 0,1, K-1)} f_{n-1} f_{n}^{K-1}\right)+\cdots \\
& \quad+h_{n}\left(a_{n(0, \ldots, 0, K-1,1)} f_{n-1}^{K-1} f_{n}+\cdots+a_{n(0, \ldots, 0,1, K-1)} f_{n-1} f_{n}^{K-1}\right)=0 .
\end{aligned}
$$

Since the product $f_{n-1}(P) f_{n}(P)$ is not identically zero on this set, then it can be used for dividing. Substituting the points $P=P_{i}^{n-1}$ and $P=P_{i}^{n}$ we obtain sets of equations of the form (19) on coefficients $a_{j(0, \ldots, 1, K-1)}$ and $a_{j(0, \ldots, K-1,1)}$. Using Lemma 2, we conclude that the matrices $\left(h_{j}\left(x, P_{i}^{k}\right)\right)_{j, i=1}^{n}$ of the corresponding systems are nondegenerated and, consequently, $a_{i(0, \ldots, 1, K-1)}=a_{i(0, \ldots, K-1,1)}=0, i=1, \ldots, n$.

Similarly, one can show that all the leading coefficients of the operators $d_{k}$ are zero. Thus, we have come to a contradiction with the fact that $K$ is the leading order of operators $d_{1}, \ldots, d_{n}$. 
To complete the proof of Theorem 1 we need

Lemma 3. The modules $M_{\Gamma}$ and $N$ coincide.

Proof. By $N(k)$ we denote the following subset

$$
N(k)=\left\{\sum_{i=1}^{n} d_{i} \psi_{i} \mid d_{i} \in \mathcal{D}_{n}, \text { ord } d_{i} \leq k-1\right\} .
$$

Since $\mathcal{D}_{n}$-module $N$ is free,

$$
\operatorname{rank}_{\mathcal{O}} N(k)=n \operatorname{rank}_{\mathcal{O}}\left\{d \psi_{1} \mid m d \in \mathcal{D}_{n}, \text { ord } d \leq k-1\right\}=n \operatorname{rank}_{\mathcal{O}} \mathcal{D}_{n}=k C_{k+n-1}^{n-1} .
$$

Consequently,

$$
\operatorname{rank}_{\mathcal{O}} N(k)=\operatorname{rank}_{\mathcal{O}} M_{\Gamma}(k) .
$$

Since there is obvious inclusion $N(k) \subseteq M_{\Gamma}(k)$, we obtain

$$
M_{\Gamma}=N .
$$

Theorem 1 is proven.

\subsection{Proof of subsidiary statements}

Choosing on $\mathbb{C} P^{1} \times \mathbb{C} P^{n-1}$ a suitable coordinate system, we assume that $\Gamma$ is obtained by the identification of hypersurfaces $(1: 0) \times \mathbb{C} P^{n-1}$ and $(0: 1) \times \mathbb{C} P^{n-1}$, i.e. $a_{2}=b_{1}=0$. Also, we can assume that $a_{1}=b_{2}=1$.

Lemma 4. Under condition (5) hypersurfaces $f=0, f_{1}=0, \ldots, f_{n}=0$ do not have common points in $\mathbb{C} P^{1} \times \mathbb{C} P^{n-1}$.

Proof. Let us recall that $f$ and $f_{1}, \ldots, f_{n}$ are the basis of the form (2), satisfying the identity (6). We find such number $k$ that $f_{k}$ satisfies $(6)$ for $c_{k} \neq 0$. Without the loss of generality, we assume $c_{n} \neq 0$. Then the systems

$$
\left\{\begin{array} { l } 
{ f = 0 , } \\
{ f _ { 1 } = 0 , } \\
{ \cdots \cdots \cdots } \\
{ f _ { n - 1 } = 0 , } \\
{ f _ { n } = 0 , }
\end{array} \quad \text { and } \quad \left\{\begin{array}{l}
f=0 \\
f_{1}-\frac{c_{1}}{c_{n}} f_{n}=0 \\
\ldots \ldots \ldots \ldots \ldots \\
f_{n-1}-\frac{c_{n-1}}{c_{n}} f_{n}=0 \\
\frac{1}{c_{n}} f_{n}=0
\end{array}\right.\right.
$$

are equivalent. It is easy to verify that $f_{k}-\frac{c_{k}}{c_{n}} f_{n}$ satisfy (3). Let $\tilde{f}_{k}=f_{k}-\frac{c_{k}}{c_{n}} f_{n}$ for $1 \leq k \leq n-1$, and $\tilde{f}_{n}=\frac{1}{c_{n}} f_{n}$.

By the definition of $f$ and condition (3), we have

$$
\begin{aligned}
f\left(z_{1}, z_{2}, t\right) & =f\left(z_{1}, 0, t\right)+f\left(0, z_{2}, t\right)=z_{1} f(1,0, t)+z_{2} f(0,1, t) \\
& =z_{1} A f(0,1, \mathcal{P}(t))+z_{2} f(0,1, t) .
\end{aligned}
$$

Since $f(z, t)$ is linear in last $n$ arguments, then the equality $f=0$ can be rewritten in the following way:

$$
f\left(0,1, z_{1} A \mathcal{P}(t)+z_{2} t\right)=0 .
$$


Likewise, we transform the equalities $\tilde{f}_{1}=0, \ldots, \tilde{f}_{n-1}=0$. We obtain the following system of linear equations

$$
f(0,1, v)=0, \quad \tilde{f}_{j}(0,1, v)=0, \quad j=1, \ldots, n-1,
$$

$v=\left(z_{1} A \mathcal{P}(t)+z_{2} t\right) \in \mathbb{C}^{n}$. By linear independence (over $\mathbb{C}$ ) of the functions $f, \widetilde{f}_{1}, \ldots, \widetilde{f}_{n-1}$ this system has the unique solution $v=0$. Thus, the equations $f=0, f_{1}=0, \ldots, f_{n-1}=0$ are equivalent to

$$
z_{1} A \mathcal{P}(t)+z_{2} t=0
$$

The solutions of this system have the form:

$$
\begin{aligned}
& \text { 1) } z_{1}=z_{2}=0 \\
& \text { 2) } t=0 \\
& \text { 3) } z_{2} /\left(A z_{1}\right)=-\lambda_{j}, \quad t=\mathbf{w}_{j} .
\end{aligned}
$$

The solutions of the form 1) and 2) do not specify any point in $\mathbb{C} P^{1} \times \mathbb{C} P^{n-1}$. Taking into account (4), case 3 ) gives $n$ different solutions. Now we add to this system the last equality $\tilde{f}_{n}=0$. According to $(7)$,

$$
\tilde{f}_{n}(z, t)=z_{1} A \tilde{f}_{n}(0,1, \mathcal{P}(t))+z_{2} \tilde{f}_{n}(0,1, t)+f(1,0, t)=\tilde{f}_{n}\left(0,1, z_{1} A \mathcal{P}(t)+z_{2} t\right)+f(1,0, t) .
$$

Taking into account (5) and 3), we have

$$
\tilde{f}_{n}(z, t)=\tilde{f}_{n}(0,1,0, \ldots, 0)+f\left(1,0, \mathbf{w}_{j}\right)=f\left(1,0, \mathbf{w}_{j}\right) \neq 0 .
$$

This reasoning ends the proving of Lemma 4.

Proof of Lemma 2. We note that since any function of the form (2) is a linear combination of the functions $f$ and $f_{1}, \ldots, f_{n}$, then from the definition of the module $M_{\Gamma}$ it follows that a different choice of the forms $f_{j}$ corresponds to a nondegenerated linear change of variables $x_{1}, \ldots, x_{n}$. This implies that if the statement of Theorem 1 is true for any fixed set $f_{1}, \ldots, f_{n}$ then it is true for any other one. Therefore, we work with easy-to-use basis.

Let us choose the basis such that the points of the intersection $P_{i}^{k}=\left(z_{i 1}^{k}, z_{i 2}^{k}, t_{i}^{k}\right)$ of the hypersurfaces $f(P)=0$ and $f_{j}(P)=0(j=1, \ldots, n ; j \neq k)$ satisfy the following two conditions:

A) first two coordinates $z_{i 1}^{k}$ and $z_{i 2}^{k}$ of the points $P_{i}^{k}$ do not vanish;

B) the set of the vectors $\left(t_{1}^{k}, \ldots, t_{n}^{k}\right) \in \mathbb{C}^{n}$ is linearly independent.

It always can be achieved. Indeed, we choose $n$ functions $\tilde{f}_{j}$ of the form (2), satisfying (3), and such that any $n-1$ from them and the function $f$ are linear independent. In the course of proving of Lemma 4 it was shown that the points of intersection of the hypersurfaces $f=0$, $\tilde{f}_{j}=0, j=1, \ldots, n ; j \neq k$, satisfy the conditions A) and B). We take one more function $\tilde{f}_{n+1}$ of the same form, satisfying (6) at $\tilde{c}_{n+1}=1$. Since the coordinates of the points of intersections continuously depend on the coefficients of the functions, then by $f_{j}$ we take $f_{j}=\tilde{f}_{j}+c_{j} \tilde{f}_{n+1}$, where $c_{j}$ are sufficiently small. From the proof of Lemma 4 we obtain that all points $P_{j}^{k}$ are different.

Let us write matrix $\left(h_{j}\left(x, P_{i}^{k}\right)\right)$ in a more convenient form. For this, we note that the condition (8) can be written as the condition on $h_{k}$

$$
h_{k}\left(x, a_{1}, b_{1}, t\right)-h_{k}\left(x, a_{2}, b_{2}, \mathcal{P}(t)\right) \Lambda A e^{-c x}=0 .
$$


Then the following equalities are true:

$$
\begin{aligned}
h_{j}\left(x, z_{1}, z_{2}, t\right) & =h_{j}\left(x, z_{1}, 0, t\right)+h_{j}\left(x, 0, z_{2}, t\right)=z_{1} \Lambda A e^{-c x} h_{j}(x, 0,1, \mathcal{P}(t))+z_{2} h_{j}(x, 0,1, t) \\
& =h_{j}\left(x, 0,1, z_{2} t+z_{1} \Lambda A e^{-c x} \mathcal{P}(t)\right) .
\end{aligned}
$$

Let $\mathbf{v}_{i}^{k}=\left(z_{i 2}^{k} t_{i}^{k}+z_{i 1}^{k} e^{-c x} \mathcal{P}\left(t_{i}^{k}\right)\right)$. Thus, the nondegeneracy condition of the matrix $\left(h_{j}\left(x, P_{i}^{k}\right)\right)$ is written in the following way

$$
\operatorname{det}\left(\begin{array}{cccc}
h_{1}\left(x, 0,1, \mathbf{v}_{1}^{k}\right) & h_{2}\left(x, 0,1, \mathbf{v}_{1}^{k}\right) & \ldots & h_{n}\left(x, 0,1, \mathbf{v}_{1}^{k}\right) \\
\vdots & \vdots & \ddots & \vdots \\
h_{1}\left(x, 0,1, \mathbf{v}_{n}^{k}\right) & h_{2}\left(x, 0,1, \mathbf{v}_{n}^{k}\right) & \ldots & h_{n}\left(x, 0,1, \mathbf{v}_{n}^{k}\right)
\end{array}\right) \neq 0 .
$$

Since $h_{j}(x, P)$ are independent over $\mathcal{O}$, from (20) we obtain that the functions $h_{j}(x, 0,1, \cdot)$, as the functions of the last $n$ arguments, are also independent over $\mathcal{O}$. Then inequality (21) is equivalent to the linear independence of the vectors $\mathbf{v}_{i}^{k}$, since $h_{i}$ are the linear forms of $\mathbf{v}_{i}^{k}$.

Let us show that the vectors $\mathbf{v}_{i}^{k}$ are linear independent. Suppose that it is wrong, i.e. there are coefficients $\gamma_{i}$ (generally speaking dependent on $x$ ) such, that $\sum \gamma_{i} \mathbf{v}_{i}^{k}=0$ or in more detail:

$$
\sum_{i=1}^{n}\left(\gamma_{i} z_{i 2} t_{i}+\gamma_{i} z_{i 1} \Lambda A e^{-c x} s_{i}\right)=0
$$

where $s_{i}^{k}=\mathcal{P}\left(t_{i}^{k}\right)$. The last equality can be written in the matrix form

$$
\left(T+\Lambda A e^{-c x} S\right) \gamma=0,
$$

where $T$ and $S$ are matrices, composed from vectors $z_{i 2} t_{i}^{k}$ and $z_{i 1} s_{i}^{k}$ respectively and independent from $x, \gamma=\left(\gamma_{1}, \ldots, \gamma_{n}\right)^{\top}$. Since the matrices $T$ and $S$ are nondegenerated, then there are not more then $n$ values $\mu_{j}$ such, that $\operatorname{det}\left(T+\mu_{j} S\right)=0$ and $\mu_{j}$ also do not depend on $x$. Consequently, for $\Lambda A e^{-c x} \neq \mu_{j}$ system (22) has only one solution $\gamma=0$, i.e., $\mathbf{v}_{i}^{k}$ are linear independent for almost every $x$ and, consequently, the determinant $\left|h_{j}\left(x, P_{i}^{k}\right)\right|$ does not vanish identically in $x$. Lemma 2 is proven.

\section{Proof of Theorem 2}

Let us choose in $M_{\Omega}(1)$ two independent over $\mathcal{O}$ functions $\varphi_{1}$ and $\varphi_{2}$

$$
\varphi_{i}=\frac{\tilde{h}_{i}(x, y, P)}{g(P)} \exp \left(x G_{1}(P)+y G_{2}(P)\right)
$$

where

$$
\tilde{h}_{i}(x, y, P)=k_{i}(x, y) z_{1} z_{2}+l_{i}(x, y) z_{1} w_{2}+m_{i}(x, y) w_{1} z_{2}+n_{i}(x, y) w_{1} w_{2}, \quad i=1,2 .
$$

The functions $\tilde{h}_{i}$ satisfy the identity

$$
\tilde{h}_{i}\left(x, y, 1: 0, t_{1}: t_{2}\right)-\tilde{h}_{i}\left(x, y, t_{1}: t_{2}, 0: 1\right) \Lambda B \exp \left(-x c_{1}-y c_{2}\right)=0 .
$$

By $P_{i}$ and $Q_{i}$ we denote the points of intersection of the curves, defined by the equations $g_{i}(P)=0$ and $g(P)=0, i=1,2$. By the infinitesimal variations $c_{1}$ and $c_{2}$, one can obtain that these points are pairwise different.

By means of direct check, we can ascertain that the determinant

$$
\operatorname{det}\left(\begin{array}{cc}
\tilde{h}_{1}\left(x, y, P_{i}\right) & \tilde{h}_{2}\left(x, y, P_{i}\right) \\
\tilde{h}_{1}\left(x, y, Q_{i}\right) & \tilde{h}_{2}\left(x, y, Q_{i}\right)
\end{array}\right)
$$


up to multiplication by nonvanishing function from $\mathcal{O}$, is equal to

$$
\frac{\sqrt{\left(\gamma \delta_{i}-\gamma_{i} \delta\right)^{2}+2 \gamma \delta c_{i}\left(\gamma \delta_{i}-\gamma_{i} \delta\right)+\delta^{2}(\gamma-2 B \delta)^{2} c_{i}^{2}}\left(\Lambda e^{-c_{1} x-c_{2} y}-1\right)^{2}}{\gamma \delta_{i}-\gamma_{i} \delta} .
$$

It is obvious that the inequality

$$
\operatorname{det}\left(\begin{array}{cc}
\tilde{h}_{1}\left(x, y, P_{i}\right) & \tilde{h}_{2}\left(x, y, P_{i}\right) \\
\tilde{h}_{1}\left(x, y, Q_{i}\right) & \tilde{h}_{2}\left(x, y, Q_{i}\right)
\end{array}\right) \neq 0
$$

is fulfilled for almost all $x$ and $y$.

Further the proof of Theorem 2 verbatim repeats the proof of Theorem 1 .

\section{$5 \quad$ Examples}

In this section we demonstrate the examples of the commuting differential operators and their common eigenvector-functions defining the basis in the free BA-modules.

\subsection{Commuting operators corresponding to the variety $\Gamma$}

Let us consider the case $n=2$. As a spectral variety we take

$$
\Gamma=\mathbb{C} P^{1} \times \mathbb{C} P^{1} /\left\{\left(1: 0, t_{1}: t_{2}\right) \sim\left(0: 1, t_{2}: t_{1}\right)\right\},
$$

i.e. in terms of Section $2 p_{1}=(1: 0), p_{2}=(0: 1), \mathcal{P}\left(t_{1}, t_{2}\right)=\left(t_{2}, t_{1}\right)$.

We introduce three functions

$$
f(P)=-z_{1} t_{1}-z_{2} t_{2}, \quad f_{1}(P)=z_{1} t_{2}+z_{2}\left(t_{1}-i t_{2}\right), \quad f_{2}(P)=-z_{1} t_{2}-z_{2}\left(t_{1}+i t_{2}\right) .
$$

Via the direct check, we can ascertain that $f(P)$ satisfies the condition (3) for $A=1$, and $f_{1}, f_{2}$ satisfy the condition (6) for $c_{1}=c_{2}=-i$.

Let us choose in $\mathcal{D}$-module $M_{\Gamma}$ the basis

$$
\begin{aligned}
& \psi_{1}(x, y, P)=\frac{z_{1} t_{1}+e^{-i(x+y)} z_{2} t_{2}}{f(P)} \exp \left(\frac{f_{1}}{f} x+\frac{f_{2}}{f} y\right), \\
& \psi_{2}(x, y, P)=\frac{z_{1} t_{2}+e^{-i(x+y)} z_{2} t_{1}}{f(P)} \exp \left(\frac{f_{1}}{f} x+\frac{f_{2}}{f} y\right) .
\end{aligned}
$$

We consider the following meromorphic functions on $\Gamma$ with the poles on the curve $f(P)=0$

$$
\lambda_{1}=\frac{2\left(z_{1} t_{2}+z_{2} t_{1}\right)}{f(P)}, \quad \lambda_{2}=\frac{i z_{1} z_{2}\left(-t_{1}^{2}+t_{2}^{2}\right)}{f(P)^{2}}, \quad \lambda_{3}=\frac{z_{1}^{2} t_{2}^{2}+3 z_{1} z_{2} t_{1} t_{2}+z_{2}^{2} t_{1}^{2}}{f(P)^{2}}
$$

Pairwise commuting operators, corresponding to these functions have the forms

$$
\begin{aligned}
D\left(\lambda_{1}\right) & =\left(\begin{array}{cc}
\partial_{x}-\partial_{y} & 0 \\
0 & \partial_{x}-\partial_{y}
\end{array}\right), \\
D\left(\lambda_{2}\right) & =\left(\begin{array}{cc}
\frac{1}{4}\left(\partial_{y}^{2}-\partial_{x}^{2}\right)+\frac{1}{2} \cot \left(\frac{x+y}{2}\right)\left(\partial_{x}-\partial_{y}\right) & \cot \left(\frac{x+y}{2}\right)-\frac{1}{2}\left(\partial_{x}+\partial_{y}\right) \\
-\frac{1}{2}\left(\partial_{x}+\partial_{y}\right) & \frac{1}{4}\left(\partial_{y}^{2}-\partial_{x}^{2}\right)
\end{array}\right) .
\end{aligned}
$$


Operator, corresponding to the function $\lambda_{3}$ has the form

$$
\begin{aligned}
& {\left[D\left(\lambda_{3}\right)\right]_{11}=\frac{1}{2} \partial_{x}^{2}+\frac{1}{2} \partial_{y}^{2}-\frac{1}{2} \cot \left(\frac{x+y}{2}\right)\left(\partial_{x}+\partial_{y}\right),} \\
& {\left[D\left(\lambda_{3}\right)\right]_{12}=0, \quad\left[D\left(\lambda_{3}\right)\right]_{21}=\frac{1}{4 \sin ^{2}\left(\frac{x+y}{2}\right)}\left(\partial_{x}-\partial_{y}\right),} \\
& {\left[D\left(\lambda_{3}\right)\right]_{22}=\frac{1}{2 \sin ^{2}\left(\frac{x+y}{2}\right)}-\frac{1}{2} \cot \left(\frac{x+y}{2}\right)\left(\partial_{x}+\partial_{y}\right)+\frac{1}{2} \partial_{x}^{2}+\frac{1}{2} \partial_{y}^{2} .}
\end{aligned}
$$

\subsection{Commuting operators corresponding to the variety $\Omega$}

Let us consider three functions

$$
\begin{aligned}
& g(P)=z_{1} w_{1}+z_{1} w_{2}+z_{2} w_{2}, \\
& g_{1}(P)=z_{1} w_{1}+2 z_{2} w_{1}-z_{2} w_{2}, \\
& g_{2}(P)=-z_{1} w_{1}+2 z_{2} w_{1}+z_{2} w_{2} .
\end{aligned}
$$

By the direct check we can ascertain that $g(P)$ satisfies the identity $(11)$ for $B=1, g_{1}(P)$ and $g_{2}(P)$ satisfy to the identity $(12)$ for $c_{1}=1$ and $c_{2}=-1$ respectively.

The curves $g(P)=0$ and $g_{1}(P)=0$ are intersected in the points

$$
P_{1}=\left(-2-\sqrt{2}: 1,-\frac{1}{\sqrt{2}}: 1\right), \quad Q_{1}=\left(-2+\sqrt{2}: 1, \frac{1}{\sqrt{2}}: 1\right),
$$

and the curves $g(P)=0$ and $g_{2}(P)=0$ are intersected in the points

$$
P_{2}=\left(-\sqrt{2}: 1,-1+\frac{1}{\sqrt{2}}: 1\right), \quad Q_{2}=\left(\sqrt{2}: 1,-1-\frac{1}{\sqrt{2}}: 1\right) .
$$

Chose the basis $\psi_{1}, \psi_{2}$ in $\mathcal{D}$-module $M_{\Omega}$

$$
\begin{aligned}
& \psi_{1}=\frac{z_{2} w_{1}}{g(P)} \exp \left(x G_{1}(P)+y G_{2}(P)\right), \\
& \psi_{2}=\frac{z_{1} w_{1} e^{y-x}+z_{1} w_{2}+z_{2} w_{2} e^{x-y}}{g(P)} \exp \left(x G_{1}(P)+y G_{2}(P)\right) .
\end{aligned}
$$

Then

$$
\begin{aligned}
& \frac{\tilde{h}_{1}\left(P_{1}, x, y\right)}{\tilde{h}_{2}\left(P_{1}, x, y\right)}=-\frac{e^{x+y}}{\sqrt{2}\left(e^{y}-e^{x}\right)\left(-e^{x}+(1+\sqrt{2}) e^{y}\right)}, \\
& \frac{\tilde{h}_{1}\left(Q_{1}, x, y\right)}{\tilde{h}_{2}\left(Q_{1}, x, y\right)}=-\frac{e^{x+y}}{\sqrt{2}\left(e^{y}-e^{x}\right)\left(e^{x}+(-1+\sqrt{2}) e^{y}\right)},
\end{aligned}
$$

thus inequality (24) is fulfilled.

Four simplest meromorphic functions on $\Omega$ with the poles on the curve $g(P)=0$ have the form

$$
\lambda_{1}=\frac{z_{2} w_{1}}{g(P)}, \quad \lambda_{2}=\frac{z_{1} z_{2} w_{1}^{2}}{g(P)^{2}}, \quad \lambda_{3}=\frac{z_{1} w_{1} z_{2} w_{2}}{g(P)^{2}}, \quad \lambda_{4}=\frac{z_{1} z_{2} w_{2}^{2}+z_{1}^{2} w_{1} w_{2}}{g(P)^{2}} .
$$

Pairwise commutating operators, corresponding to these functions have the form

$$
D\left(\lambda_{1}\right)=\left(\begin{array}{cc}
\frac{1}{4}\left(\partial_{x}+\partial_{y}\right) & 0 \\
0 & \frac{1}{4}\left(\partial_{x}+\partial_{y}\right)
\end{array}\right),
$$




$$
\begin{aligned}
{\left[D\left(\lambda_{2}\right)\right]_{11}=} & \frac{e^{x}}{8\left(e^{x}-e^{y}\right)}\left(\partial_{x}^{2}-\partial_{y}^{2}\right)-\frac{e^{x+y}}{4\left(e^{x}-e^{y}\right)^{2}}\left(\partial_{x}+\partial_{y}\right), \\
{\left[D\left(\lambda_{2}\right)\right]_{12}=} & \frac{e^{x+y}}{16\left(e^{x}-e^{y}\right)^{2}}\left(\partial_{x}+\partial_{y}\right)^{2}, \\
{\left[D\left(\lambda_{2}\right)\right]_{21}=} & \frac{1}{8}\left(e^{y-x}-e^{x-y}-2\right) \partial_{x}^{2}+\frac{1}{8}\left(e^{x-y}-e^{y-x}-2\right) \partial_{y}^{2}+\frac{1}{2} \partial_{x} \partial_{y} \\
& +\frac{e^{x}+e^{2 x-y}+5 e^{y}-e^{2 y-x}}{4\left(e^{x}-e^{y}\right)} \partial_{x}+\frac{3 e^{x}-e^{2 x-y}+3 e^{y}+e^{2 y-x}}{4\left(e^{y}-e^{x}\right)} \partial_{y}-\frac{e^{y}\left(2 e^{x}+e^{y}\right)}{\left(e^{x}-e^{y}\right)^{2}}, \\
{\left[D\left(\lambda_{2}\right)\right]_{22}=} & \frac{e^{x}}{8\left(e^{y}-e^{x}\right)} \partial_{x}^{2}-\frac{1}{4} \partial_{x} \partial_{y}+\frac{e^{x}-2 e^{y}}{8\left(e^{y}-e^{x}\right)} \partial_{y}^{2}+\frac{e^{y}\left(2 e^{x}+e^{y}\right)}{8\left(e^{x}-e^{y}\right)^{2}}\left(\partial_{x}+\partial_{y}\right) .
\end{aligned}
$$

The operator, corresponding to the function $\lambda_{3}$ has the form

$$
\begin{aligned}
{\left[D\left(\lambda_{3}\right)\right]_{11}=} & \frac{\left(e^{x}+e^{y}\right)}{8\left(e^{y}-e^{x}\right)}\left(\partial_{x}^{2}-\partial_{y}^{2}\right)+\frac{\left(e^{2 x}+e^{2 y}\right)}{4\left(e^{y}-e^{x}\right)^{2}}\left(\partial_{x}+\partial_{y}\right), \\
{\left[D\left(\lambda_{3}\right)\right]_{12}=} & \frac{e^{x+y}}{8\left(e^{y}-e^{x}\right)^{2}}\left(\partial_{x}-\partial_{y}\right)^{2}, \\
{\left[D\left(\lambda_{3}\right)\right]_{21}=} & \frac{1}{4}\left(2+e^{x-y}-e^{y-x}\right) \partial_{x}^{2}-\partial_{x} \partial_{y}+\frac{1}{4}\left(2-e^{x-y}+e^{y-x}\right) \partial_{y}^{2} \\
& +\frac{2 e^{x}+e^{2 x-y}+4 e^{y}-e^{2 y-x}}{2\left(e^{y}-e^{x}\right)} \partial_{x}+\frac{4 e^{x}-e^{2 x-y}+2 e^{y}+e^{2 y-x}}{2\left(e^{x}-e^{y}\right)} \partial_{y} \\
& +\frac{e^{2 x}+e^{2 y}+4 e^{x+y}}{\left(e^{x}-e^{y}\right)^{2}}, \\
{\left[D\left(\lambda_{3}\right)\right]_{22}=} & \frac{3 e^{x}-e^{y}}{8\left(e^{x}-e^{y}\right)} \partial_{x}^{2}+\frac{1}{2} \partial_{x} \partial_{y}+\frac{e^{x}-3 e^{y}}{8\left(e^{x}-e^{y}\right)} \partial_{y}^{2}-\frac{3 e^{x+y}}{2\left(e^{y}-e^{x}\right)^{2}}\left(\partial_{x}+\partial_{y}\right) .
\end{aligned}
$$

Operator corresponding to the function $\lambda_{4}$ has the form

$$
\begin{aligned}
{\left[D\left(\lambda_{4}\right)\right]_{11}=} & \frac{e^{x}+3 e^{y}}{4\left(e^{x}-e^{y}\right)} \partial_{x}^{2}+\frac{1}{2} \partial_{x} \partial_{y}-\frac{3 e^{x}+e^{y}}{4\left(e^{x}-e^{y}\right)} \partial_{y}^{2} \\
& -\frac{e^{2 x}+3 e^{2 y}}{2\left(e^{x}-e^{y}\right)^{2}} \partial_{x}-\frac{3 e^{2 x}+e^{2 y}}{2\left(e^{x}-e^{y}\right)^{2}} \partial_{y}-\frac{2 e^{x+y}}{\left(e^{y}-e^{x}\right)^{2}} \\
{\left[D\left(\lambda_{4}\right)\right]_{12}=} & \frac{e^{x+y}}{2\left(e^{y}-e^{x}\right)^{2}}\left(\left(\partial_{x}+\partial_{y}\right)^{2}+\partial_{x}+\partial_{y}\right), \\
{\left[D\left(\lambda_{4}\right)\right]_{21}=} & \left(e^{y-x}-e^{x-y}-2\right) \partial_{x}^{2}+4 \partial_{x} \partial_{y}+\left(e^{x-y}-e^{y-x}-2\right) \partial_{y}^{2} \\
& +\frac{5 e^{x}+e^{2 x-y}+9 e^{y}-3 e^{2 y-x} \partial_{x}+\frac{9 e^{x}-3 e^{2 x-y}+5 e^{y}+e^{2 y-x}}{e^{x}-e^{y}} \partial_{y}}{\left(e^{x}-e^{y}\right)^{2}} \\
& +\frac{2 e^{-x-y}\left(e^{4 x}+e^{4 y}-6 e^{2(x+y)}-4 e^{3 x+y}-4 e^{x+3 y}\right)}{\left[D\left(\lambda_{4}\right)\right]_{22}=} \\
& \frac{7 e^{x}-3 e^{y}}{4\left(e^{y}-e^{x}\right)} \partial_{x}^{2}-\frac{3}{2} \partial_{x} \partial_{y}+\frac{3 e^{x}-7 e^{y}}{4\left(e^{y}-e^{x}\right)} \partial_{y}^{2} \\
& -\frac{e^{2 x}+3 e^{2 y}-16 e^{x+y}}{2\left(e^{x}-e^{y}\right)^{2}} \partial_{x}-\frac{3 e^{2 x}+e^{2 y}-16 e^{x+y}}{2\left(e^{x}-e^{y}\right)^{2}} \partial_{y} .
\end{aligned}
$$




\section{A Structures of algebraic varieties on $\Gamma$ and $\Omega$}

We show that on $\Gamma$ and $\Omega$ structures of algebraic varieties can be introduced. For this in the first case we construct a smooth morphism from $\mathbb{C} P^{1} \times \mathbb{C} P^{n-1}$ to $\mathbb{C} P^{2} \times \mathbb{C} P^{2 n-1}$, and in the second case from $\mathbb{C} P^{1} \times \mathbb{C} P^{1}$ to $\mathbb{C} P^{11}$. The morphisms are injective everywhere except gluing hypersurfaces. The images of the morphisms are algebraic varieties which define required structures on $\Gamma$ and $\Omega$.

\section{A.1 Variety $\Gamma$}

By choosing the convenient coordinate system on $\mathbb{C} P^{1}$ we can assume that

$$
\Gamma=\mathbb{C} P^{1} \times \mathbb{C} P^{n-1} /\{(1: 0, t) \sim(0: 1, \mathcal{P}(t))\} .
$$

We consider the mapping

$$
\varphi_{1}: \mathbb{C} P^{1} \times \mathbb{C} P^{n-1} \rightarrow \mathbb{C} P^{2} \times \mathbb{C} P^{2 n-1},
$$

defined by the formula:

$$
\varphi_{1}(z, t)=(u, v),
$$

where $u=\left(u_{1}: u_{2}: u_{3}\right)$,

$$
u_{1}=z_{1}^{2} z_{2}, \quad u_{2}=z_{1} z_{2}^{2}, \quad u_{3}=z_{1}^{3}+z_{2}^{3}, \quad v=\left(z_{1}^{2} t+z_{2}^{2} \mathcal{P}^{-1}(t): z_{1} z_{2} t\right) .
$$

Here $v=\left(\xi_{1}: \cdots: \xi_{n}: \eta_{1}: \cdots: \eta_{n}\right), \xi_{j}=z_{1} 2 t_{j}+z_{2} 2 r_{j}$ and $\eta_{j}=z_{1} z_{2} t_{j}$, where $r_{j}$ is $j$-th coordinate $\mathcal{P}^{-1}(t), j=1, \ldots, n$.

Lemma 5. The mapping $\varphi_{1}$ is correctly defined on $\Gamma$ and is the embedding of $\Gamma$. The image of $\varphi_{1}$ is defined by the equations:

$$
\begin{aligned}
& u_{1}^{3}+u_{2}^{3}=u_{1} u_{2} u_{3}, \\
& u_{1}^{2} \eta+u_{2}^{2} \mathcal{P}^{-1}(\eta)=u_{1} u_{2} \xi, \\
& u_{3} \eta+u_{1} \mathcal{P}(\eta)+u_{2} \mathcal{P}^{-1}(\eta)=u_{1} \xi+u_{2} \mathcal{P}(\xi) .
\end{aligned}
$$

Proof. Let us show that the image of the point $(z, t) \in \mathbb{C} P^{1} \times \mathbb{C} P^{n-1}$ satisfies the equations (25)-(27). The equalities (25) and (26) obviously follow from the definition of $\varphi_{1}$. The equality (27) for $z_{1} \neq 0$ and $z_{2} \neq 0$ is the corollary of (25) and (26). Indeed, from (26) we obtain

$$
u_{1}^{2} \mathcal{P}(\eta)+u_{2}^{2} \eta=u_{1} u_{2} \mathcal{P}(\xi) .
$$

Let us multiply the obtained equality by $u_{2}$, equality (26) by $u_{1}$ and take a sum. Dividing the result by $u_{1} u_{2} \neq 0$, we obtain (27). If $z_{1}$ or $z_{2}$ are equal to zero, then $\eta=0$, and, consequently, the left and right parts of (27) vanish.

We show that for any point $(u, v) \in \mathbb{C} P^{2} \times \mathbb{C} P^{2 n-1}$, satisfying (25)-(27), the inverse image can be found. Note that from $(25)$ it follows that $u_{1}$ and $u_{2}$ can vanish only simultaneously.

If $u_{1}=u_{2}=0$, then from (27) we obtain $\eta=0$ and the inverse image can be either point $(1: 0, \xi)$, or point $(0: 1, \mathcal{P}(\xi))$, which are identified in $\Gamma$.

Now we consider the case $u_{1} \neq 0$ and $u_{2} \neq 0$. From (26) it follows that $\xi \neq 0$. In this case as the inverse image of the point we can take point $\left(u_{1}: u_{2}, \eta\right)$. By easy calculations it can be checked that $\varphi_{1}\left(u_{1}: u_{2}, \eta\right)=\left(u_{1}: u_{2}: u_{3}, \xi: \eta\right)$. If any other point $B=\left(a: b, s_{1}: \cdots: s_{n}\right)$ is the inverse image of $\left(u_{1}: u_{2}: u_{3}, \xi: \eta\right)$, then $a: b=u_{1}: u_{2}$ and, consequently, $a \neq 0, b \neq 0$, $\eta_{j}=a b s_{j}$, i.e. $B=\left(u_{1}: u_{2}, \eta\right)$, which shows injectivity of the mapping $\varphi_{1}$ on $\Gamma$.

By direct calculations one can show that the differential of the mapping $\varphi_{1}$ is nondegenerated. 


\section{A.2 Variety $\Omega$}

Let us consider the mapping

$$
\varphi_{2}: \mathbb{C} P^{1} \times \mathbb{C} P^{1} \rightarrow \mathbb{C} P^{11},
$$

defined by the formula:

$$
\varphi_{2}\left(z_{1}: z_{2}, w_{1}: w_{2}\right)=\left(u_{1}: \cdots: u_{12}\right),
$$

where

$$
\begin{aligned}
& u_{1}=z_{1}^{3}\left(w_{1}^{3}+w_{2}^{3}\right)+\left(z_{2} w_{2}\right)^{3}, \quad u_{2}=z_{1}^{3} w_{1}^{2} w_{2}+z_{1}^{2} z_{2} w_{2}^{3}, \quad u_{3}=z_{1}^{3} w_{1} w_{2}^{2}+z_{1} z_{2}^{2} w_{2}^{3}, \\
& u_{4}=z_{1}^{2} z_{2} w_{1}^{3}, \quad u_{5}=z_{1}^{2} z_{2} w_{1}^{2} w_{2}, \quad u_{6}=z_{1}^{2} z_{2} w_{1} w_{2}^{2}, \quad u_{7}=z_{1} z_{2}^{2} w_{1}^{3}, \quad u_{8}=z_{1} z_{2}^{2} w_{1}^{2} w_{2}, \\
& u_{9}=z_{1} z_{2}^{2} w_{1} w_{2}^{2}, \quad u_{10}=z_{2}^{3} w_{1}^{3}, \quad u_{11}=z_{2}^{3} w_{1}^{2} w_{2}, \quad u_{12}=z_{2}^{3} w_{1} w_{2}^{2} .
\end{aligned}
$$

We can easily ascertain that $u_{j}$ do not vanish simultaneously (for example, $u_{1}, u_{2}, u_{3}$ and $u_{10}$ equal zero if and only if $z_{1}=z_{2}=0$ or $w_{1}=w_{2}=0$ ).

Lemma 6. The mapping $\varphi_{2}$ is correctly defined on $\Omega$ and is the embedding of $\Omega$.

Proof. We show that the mapping $\varphi_{2}$ identifies only points $\left(1: 0, t_{1}: t_{2}\right)$ and $\left(t_{1}: t_{2}, 0: 1\right)$ on $\mathbb{C} P^{1} \times \mathbb{C} P^{1}$.

If $u_{10} \neq 0$, then from the definition of $\varphi_{2}$ it follows that the inverse image has the form $\left(z_{1}: z_{2}, w_{1}: w_{2}\right)=\left(u_{7}: u_{10}, u_{10}: u_{11}\right)$.

If $u_{10}=0$, then $u_{k}=0$ for $4 \leq k \leq 12, u_{2}$ and $u_{3}$ vanish simultaneously. Two cases are possible:

a) $u_{2} \neq 0$ and $u_{3} \neq 0$, then inverse image is one of the two points $\left(1: 0, u_{2}: u_{3}\right)$ or $\left(u_{2}: u_{3}, 0: 1\right)$, which are identified in $\Omega$;

b) $u_{2}=u_{3}=0$, then $u_{1} \neq 0$ and inverse image is one of the three points $(1: 0,1: 0)$, $(1: 0,0: 1)$ or $(0: 1,0: 1)$, which are also identified in $\Omega$.

By direct calculations one can show that the differential of the mapping $\varphi_{2}$ is nondegenerated.

\section{Acknowledgements}

The work is supported by the Program of Russian Academy of Sciences "Fundamental Problems of Nonlinear Dynamics". The second author (A.E.M) is thankful to Atsushi Nakayashiki for the invitations in Kyushu University, useful discussions of our results. The second author is also grateful to Grant-in-Aid for Scientific Research (B) 17340048 for financial support of the visits in Kyushu University.

\section{References}

[1] Nakayashiki A., Structure of Baker-Akhiezer modules of principally polarized Abelian varieties, commuting partial differential operators and associated integrable systems, Duke Math. J. 62 (1991), 315-358.

[2] Nakayashiki A., Commuting partial differential operators and vector bundles over Abelian varieties, Amer. J. Math. 116 (1994), 65-100.

[3] Mironov A.E., Commutative rings of differential operators corresponding to multidimensional algebraic varieties, Siberian Math. J. 43 (2002), 888-898, math-ph/0211006.

[4] Rothstein M., Sheaves with connection on Abelian varieties, Duke Math. J. 84 (1996), 565-598, alg-geom/9602023.

[5] Rothstein M., Dynamics of the Krichever construction in several variables, J. Reine Angew. Math. 572 (2004), 111-138, math.AG/0201066.

[6] Previato E., Multivariable Burchnall-Chaundy theory, Philos. Trans. R. Soc. Lond. Ser. A Math. Phys. Eng. Sci. 336 (2008), 1155-1177. 